\title{
Aproveitamento de Resíduos Industriais de Alimentos com Potencial Aplicação em Cosméticos Naturais
}

\author{
Nathalia P. Barbosa \& Edemilson C. da Conceição
}

Os resíduos agroindustriais de alimentos representa uma preocupação mundial e vem causando problemas ambientais, sociais e econômicos a todos envolvidos. Este trabalho tem como objetivo revisar aspectos relacionados aos resíduos industriais e sua aplicação em cosméticos. É notória a necessidade por novas aplicações dos resíduos de alto valor agregado para indústria. $\mathrm{O}$ segmento de cosmético cada vez mais crescente e desafiado por inovações, inclusive sociais e ambientais, representa uma aplicação de alto impacto para esses rejeitos ricos em nutrientes, bioativos e vitaminas capazes de apresentar atividades biológicas significativas no desenvolvimento de novos produtos, sendo estes produtos de valoração mundial.

Palavras-chave: subprodutos; inovação.

The agro-food waste is a global concern and has caused environmental, social and economic problems to all Involved. This work aims to review aspects related to industrial waste and its application in cosmetics . It is evident the need for new applications of waste with high value added for industry. The cosmetics segment increasingly growing and challenged by innovations, including social and environmental, represents an application of high impact to these rich tailings in nutrients, bioactive and vitamins able to have significant biological activity in developing new products for the world.

Keywords: byproducts; innovation. 


\section{Introdução}

Com a crescente produção de alimentos, verifica-se o aumento da geração de resíduos e diversos estudos em todo o mundo estão sendo realizados a fim de criar soluções para diminuir ou eliminar os passivos ambientais gerados pelas agroindústrias de alimentos (ALBURQUERQUE et al., 2012; VIRMOND et al., 2012; CASTRO; SATO, 2013) $)^{1,2,3}$. Entretanto, para que os resíduos sejam aproveitados e apresentem maior valor agregado é necessário o conhecimento da composição química a partir de investigações científicas e tecnológicas (HOFFMANN et al., 2009) . $^{4}$

Esses resíduos possuem em sua composição vitaminas, minerais, fibras e compostos antioxidantes importantes para as funções fisiológicas. No entanto, na maioria das agroindústrias, são descartados (MATIAS et al., 2005) Tais resíduos poderiam ser utilizados, minimizando o desperdício de alimentos e gerando uma nova fonte alimentar ou novos produtos, como por exemplo, na área de cosméticos. As frutas são ricas em muitos nutrientes e compostos antioxidantes, e esses constituintes são responsáveis pelas atividades biológicas dos produtos cosméticos (MELO et al., 2010)

Segundo Silva e Halberstadt (2012) ${ }^{7}$, as empresas que buscarem a sustentabilidade em seus negócios ganharão espaço de mercado, agregarão valor aos processos e à competitividade. Atualmente, além da lucratividade, é necessário que os empresários de todos os setores se atentem aos aspectos sociais e ambientais (SILVA; HALBERSTADT, 2012) ${ }^{7}$. Afinal, os produtores e fabricantes têm a responsabilidade tanto sobre o produto, mesmo após o seu período de vida útil, quanto pelos resíduos gerados

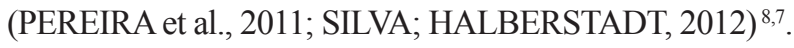

No caso específico de cosméticos naturais ou à base de produtos naturais, o mercado encontra-se em expansão. Mesmo diante da constatação da quantidade disponibilidade de matéria-prima agroindustrial, ainda não é comum encontrar no mercado produtos dessa categoria que utilizassem, como base de seus produtos, tal matéria. Avaliando o estado da arte do resíduo, verifica-se que, até então, apenas estudos relacionados à composição química foram realizados, sendo testes físico-químicos e ensaios do potencial antioxidante do subproduto, e estes apresentaram resultados relevantes (KOBORI; JORGE, 2005; AMIM; MUKHRIZAH, 2006; KONG; ISMAIL, 2011) ${ }^{9,10,11 .}$
O setor de higiene pessoal, perfumaria e cosméticos no Brasil representa um mercado que nos últimos 15 anos apresenta um crescimento significativo e hoje é o $3^{\circ}$ maior do mundo. Sobretudo na área de produtos que visem cuidar da pele, não só apostando na manutenção e recuperação de uma pele saudável e com boa aparência, como também na prevenção de possíveis alterações que a pele está sujeita (ABIHPEC, 2014) ${ }^{12}$.

A busca por inovações no segmento de higiene pessoal, perfumaria e cosmética é necessária e contínua para se manterem no mercado. As atividades de P\&D, objetivando desenvolvimento de inovações ou a instalação de novos processos vinculados ao aproveitamento dos resíduos das agroindústrias de alimentos, é iniciante no país e merece valoração pelo impacto gerado. A obtenção de um material sustentável e rico em nutrientes é fundamental para melhor destinação dos resíduos da produção industrial de alimentos e contribui com inovações em outros segmentos, como o de cosméticos, cada vez mais crescente no mundo.

\section{Fontes dos Dados}

Para a realização desse trabalho, buscaramse artigos originais e revisões indexados nas bases Periódicos Capes, Science Direct, Scifinder e Scielo. Utilizaram-se as palavras-chave: resíduos, cosméticos e inovação (português e inglês). Buscaram-se ainda dados importantes contidos em notícias nacionais e internacionais que tratam de assuntos, tais como química de produtos naturais e sustentabilidade. Não houve restrição no que se refere ao ano de publicação.

\section{Resíduos Agroindustriais}

Segundo Crittenden e Kolaczkowski (1995) ${ }^{13}$, por definição resíduo, no processo industrial, é tudo que não é considerado matéria-prima segundo as especificações. $\mathrm{Ou}$ ainda, insumos, subprodutos ou produto que requer tempo e capital para seu gerenciamento. Várias indústrias geram quantidades significativas de resíduos que prejudicam o meio ambiente, tais como indústria têxtil, de borracha, papel, plásticos, couro, cosméticos, farmacêutica e, principalmente, a de alimentos. A crescente preocupação com o meio ambiente abrange todos os segmentos industriais na busca de alternativas para o correto aproveitamento dos rejeitos (HU; CHEN; YUAN, 2010) ${ }^{14}$. 
Tratando-se de políticas para evitar a disposição de resíduos pelas agroindústrias no país, foi criada a Lei $\mathrm{n}^{\circ}$ 12.305/10 que institui a Política Nacional de Resíduos Sólidos (PNRS), as quais dispõem sobre a prevenção e a redução na geração de resíduos. As indústrias têm o prazo até 2014 para apresentarem o correto destino para seus rejeitos industriais (BRASIL, 2010) ${ }^{15}$.

A produção das agroindústrias brasileiras exige, cada vez mais, técnicas mais desenvolvidas para o tratamento dos resíduos eliminados. O passivo ambiental gerado pelas indústrias de alimentos é um resíduo com grande potencial de reutilização e devemapresentar um destino adequado, pois além de gerar impactos ambientais negativos, como problemas ambientais como proliferação de insetos e pragas, consequentemente, possíveis transtornos na qualidade dos produtos oferecidos. Entretanto, pode constituir matérias-primas ricas em nutrientes e energia, e exigem investimentos em tratamentos para controlar a poluição (PELIZER; PONTIERI; MORAES, 2007) ${ }^{16 .}$

\section{Cosméticos}

De acordo com a definição conferida pela legislação vigente, Cosméticos, Produtos de Higiene e Perfumes "são preparações constituídas por substâncias naturais ou sintéticas, de uso externo nas diversas partes do corpo humano, pele, sistema capilar, unhas, lábios, órgãos genitais externos, dentes e membranas mucosas da cavidade oral, com o objetivo exclusivo ou principal de limpá-los, perfumá-los, alterar sua aparência e ou corrigir odores corporais e ou protegê-los ou mantê-los em bom estado" (BRASIL, 2004) ${ }^{17}$. Milhões de consumidores usam produtos de higiene pessoal e cosméticos (HPPC) e os seus ingredientes, numa base diária (ABIHPEC, 2014; NOHYNEK et al., 2010) ${ }^{12,18}$.

Os cosméticos são aplicados principalmente na pele, cabelo e unhas. São produtos utilitários usados extensivamente em todo o mundo para manter $\mathrm{e}$ melhorar o aspecto geral do rosto e outras partes do corpo, por exemplo, boca, dedo da mão, olho, cabelo, etc. (PAREEK et al., 2012) ${ }^{19}$. Produtos para cuidados pessoais incluem uma grande variedade de produtos e tipos de formulação, tais como sabonetes, shampoo e produtos de higiene pessoal, protetores solares, pele e cabelos cuidados produtos, tinturas de cabelo, maquiagens, batons, cremes dentais, produtos de higiene dental, desodorantes, pessoal produtos de higiene e muitos outros (ANTIGNAC, 2011) ${ }^{20}$.

Muitas substâncias de origem botânica, animal, ou de fontes de síntese química, são testados ou investigados como ativos em cosméticos (GAO et al., 2008) ${ }^{21}$. Durante as últimas décadas, tem havido um aumento no uso de produtos naturais em cosméticos. Utilizam uma variedade de princípios ativos de várias plantas, incluindo vitaminas, flavonoides, enzimas, taninos, ácidos de fruta, aminoácidos, açúcares, glicosídeos, óleos essenciais e corante (PAREKK, 2012) ${ }^{19}$. Ingredientes cosméticos botânicos incluem aquoso ou não-aquoso extratos vegetais, tinturas, óleos ou óleos essenciais, ingredientes de plantas purificados. A variedade de plantas e alimentos que fornecem estes ingredientes é grande, variando a partir de fontes de alimentos básicos, como cereais, frutas e legumes, a ervas usadas na medicina tradicional (ALLEMANN e BAUMANN, 2009) ${ }^{22}$.

$\mathrm{O}$ principal fator para o mercado crescente por matérias-primas de origem natural é a demanda dos consumidores por produtos que são percebidos quanto mais saudáveis, orgânicos e ecológicos (ALLEMANN e BAUMANN, 2009) ${ }^{22}$. Nos últimos anos, a indústria de alimentos e saúde nacional / internacional e autoridades de segurança alimentar foram questionados pela organização, segurança e avaliação de aproveitamento de rejeitos como novos ingredientes derivados de alimentos para a utilização em cosméticos. Essas discussões consistentemente produziu um consenso que as especificações adequadas de identidade e composição química são as questõeschave da avaliação de segurança de ingredientes de origem vegetal, ainda carente de estudos científicos (ANTIGNAC, 2011) ${ }^{20}$.

\section{Desafios Tecnológicos}

Alimentos e cosméticos, já por alguns anos, representam uma tendência de significativa potencialidade de mercado. A indústria do segmento higiene pessoal, perfumaria e cosméticos não visa somente à beleza, mas principalmente, busca propiciar com seus produtos a total saúde da pele. Associado a esse fator, verifica-se cada vez mais uma preocupação da academia e o meio empresarial com a utilização de substâncias bioativas naturais em produtos cosméticos (WANG, 2015) ${ }^{23}$. 
A exploração de subprodutos do processamento de frutas e vegetais, como fonte de compostos funcionais e sua aplicação em cosméticos, é um campo promissor, devido à grande variedade de espécies vegetais contendo categorias de compostos com atividades biológicas comprovadas. Esses subprodutos são parcialmente aproveitados na fabricação de ração animal, mas a maior parte do resíduo é descartada ou utilizada em compostagem.

Existe a necessidade de explorar um possível aproveitamento de resíduos provenientes da agroindústria, com a finalidade de diminuir o impacto ambiental ocasionado pelo descarte deste subproduto e consequentemente, atender às Boas Práticas de Fabricação na Indústria de Alimentos exigidas pela legislação sanitária, ambiental e do mercado consumidor. Além disso, o conhecimento do valor nutricional dos subprodutos contribui para agregar valor à cadeia produtiva desse fruto e ainda, contribuir com novas frentes de mercado industrial.

Outro ponto importante consiste no aumento do interesse por cosméticos à base de produtos naturais. Hoje, há uma crescente demanda dos consumidores por cosméticos contendo ingredientes naturais e / ou orgânicos. Em resposta, tem sofrido um crescimento significativo nas vendas de cosméticos natural e orgânico em supermercados, farmácias, salões de beleza e farmácias em todo o mundo, especialmente na América do Norte e Europa Ocidental (ANTIGNAC, 2011) ${ }^{20}$. De acordo com um novo relatório de mercado publicado pela Transparency Market Research a demanda global por produtos de higiene pessoal orgânicos foi de US \$ 7,6 bilhões em 2012 deve chegar a 13,2 bilhões dólares americanos em 2018, crescendo a uma taxa anual média de 9,6\% 2012-2018 (PENNING, 2013) ${ }^{24}$.

\section{Conclusão e Considerações Finais}

Assim, de acordo com as elevadas taxas de produção de resíduos agroindustriais, gerados a partir do processamento da goiaba; a importância dos ativos biológicos para saúde; a manutenção da beleza da população e considerando o crescimento emergente do setor de produtos de Higiene Pessoal, Perfumaria e Cosméticos, considera-se relevante a pesquisa e o desenvolvimento de cosméticos elaborados a partir de subprodutos da agroindústria de alimentos.

Torna-se emergente a prospecção tecnológica e a exploração econômica dos resíduos agroindustriais, que acenam com amplo espectro de uso e que ainda não estão sendo exploradas comercialmente em todo o seu potencial. Neste contexto, a obtenção, a caracterização do subproduto, pesquisa e avaliação do potencial das atividades biológicas surgem como estudos iniciais em resíduos agroindustriais que poderão ser aplicados na área cosmética.

Assim, agregar valor a subprodutos ricos em nutrientes essenciais, com apelo de mercado, tem grande importância para o desenvolvimento da indústria brasileira de farmoquímicos, cosmética e de alimentos. Os processos para a total utilização dos subprodutos resultantes do processamento de alimentos em larga escala e de forma viável devem ser desenvolvidos e otimizados, contribuindo de forma decisiva para o setor de alimentos e cosméticos, resultando em produção sustentável e inovadora.

\section{Referências}

1. Alburquerque, J.A.; Fuente, C.D.L.; Ferrer-Costa, A.; Carrasco L.; Cegarra, J.; Abad, M.; Bernal, M.P.. Assessment of the fertiliser potential of digestates from farm and agroindustrial residues. Biomass and Bioenergy. 2012, 40, 181-189.

2. Virmond, E.; De Sena, R.F.; Albrecht, W.; Althoff, C.A.; Moreira, R.F.P.M.; José, H.J. Characterisation of agroindustrial solid residues as biofuels and potential application in thermochemical processes. Waste Management, 2012, 32, 1952-1961.

3. Castro, R.J.S.; Sato, H.H.. Synergistic effects of agroindustrial wastes on simultaneous production of protease and $\alpha$-amylase under solid state fermentation using a simplex centroid mixture design. Industrial Crops and Products. 2013, 49, 813-821.

4. Hoffmann-Ribani, R.; Huber, L. S.; Rodriguez-Amaya, D.B. Flavonols in fresh and processed brazilian fruits. Journal of Food Composition and Analysis. 2009, 22, 263-268.

5. Matias, M.F.O.; Oliveira, E.L.; Gertrudes, E.; Magalhâes, M.A. Use of fibres obtained from the cashew (Anacardium ocidentale, L) and guava (Psidium guajava) fruits for enrichment of food products. Brazilian Archives of Biology and Technology, Curitiba. 2005, 48, 143-150.

6. Melo, P.S. Composição química e atividade biológica de resíduos agroindustriais. Dissertação (Mestrado) Escola Superior de Agricultura “Luiz de Queiroz", ESAL/USP, 
Piracicaba, 2010.

7. Silva, V.A.; Halberstadt, K.F. Sustainable waste allocation of production chain of agroindustries. $1^{\circ}$ Fórum internacional ecoinovar, Santa Maria/RS, 2012.

8. Pereira, F.M.; Kavati, R. Contribuição da pesquisa científica brasileira no desenvolvimento de algumas frutíferas de clima subtropical. Rev. Bras. Frutic. Jaboticabal. 2011, 33.

9. Kobori, C.N.; Jorge, N. Characterization of some seed oils of fruits for utilization of industrial residues. Ciênc. agrotec., Lavras. 2005, 29, 5, 1008-1014.

10. Amim, I.; Mukhrizah, O. Antioxidant capacity of methanolic and water extracts prepared from food-processing by-products. Journal of the Science of Food and Agriculture. 2006, 86, 5, 778-784.

11. Kong, K.W.; Ismail, A. Lycopene content and lipophilic antioxidant capacity of by-products from Psidium guajava fruits produced during puree production industry. Food and Bioproducts Processing, 2011, 89, 53-61.

12. Abihpec, Associação Brasileira da Indústria de Higiene Pessoal, Perfumaria e Cosméticos. III Caderno de Tendências, 2014-2015.

13. Crittenden, B.; Kolaczkowski, S. Waste minimization: a practical guide. England: IChemE, 1995, 81.

14. Hu, Z.; Chen, H.; Yuan, J.S. Removal of Congo red from aqueous solution by cattail root. J. Hazard. Mater. 2010, 173, 292-297.

15. Brasil, Lei ${ }^{\circ} 12.305,2$ de agosto de 2010. Institui a Política Nacional de Resíduos Sólidos, 2010.

16. Pelizer, L. H.; Pontieri, M. H.; Moraes, I. de O. Utilização de resíduos agro-industriais em processos biotecnológicos como perspectiva de redução do impacto ambiental. Journal of Technology Management \& Innovation, 2007, 2, 118-127.

17. Brasil, Agência Nacional de Vigilância Sanitária (ANVISA). Diário Oficial da União. Resolução RDC n 306, de 07 de dezembro de $\mathbf{2 0 0 4}$.

18. Nohynek, G.J.; Antignac, E.; RE, T.; Toutain, H. Safety assessment of personal care products/cosmetics and their ingredientes. Toxicology and Applied Pharmacology. 2010, 243, 239-259.

19. Pareek, A.; Jain, V.; Ratan, Y.; Sharma, S.; Jain, P.K.; Dave, V. Mushrooming of Herbal's in New Emerging Market of Cosmaceuticals. , International Journal of Advanced in Pharmaceutical \& Bio Science. 2012, 2 (4), 473-480.

20. Antignac, E.; Nohynek, G.J.; Re, T.; Clouzeau, J.; Toutain, $\mathrm{H}$. Safety of botanical ingredients in personal care products/ cosmetics. Food and Chemical Toxicology. 2011, 49, 324-341.

21. Gao, Xing-Hua; Li Zhang; Huachen Wei; Hong-Duo Chen. Efficacy and safety of innovative cosmeceuticals. Clinics in Dermatology. 2008, 26, 367-374.
22. Allemann, I.B., Baumann, L. Botanicals in skin care products. Internat. J. Dermatol. 2009, 48, 923-934.

23. Wang, H. M. D. Recent Advancements in Cosmetics and Food from bioactive Natural Products and Chemical Synthetic Compounds. Current Organic Chemistry, 2015, 19, 1.

24. Penning, A. Demand for Organic Beauty to Grow to Over $\$ 13$ Billion by 2018, Report Says. Cosmetics \& Toiletries, Science Applied. 2013, 26

\section{Nathalia Pedroso Barbosa ${ }^{\text {* }}$ e Edemilson Cardoso da Conceição}

\author{
${ }^{1}$ Instituto SENAI de Tecnologia em Alimentos e Bebidas,- Rua \\ Professor Lázaro Costa nº 348, Vila Canaã, CEP: 74415-420, Goiânia, \\ Goiás. \\ ${ }^{2}$ Universidade Federal de Goiás, Faculdade de Farmácia, Laboratório \\ de PD\&I em Bioprodutos, Praça Universitária, n 1116 , Setor Leste \\ Universitário, Goiânia, Goiás. \\ "E-mail: nathaliaquifarma@gmail.com
}

